\title{
ERROS DE MEDICAÇÃO: análise do conhecimento da equipe de enfermagem de uma instituição hospitalar
}

\author{
Paulo Celso Prado T EL LES FIL H Oa, M arcus Fernando da Silva PRAXEDES, \\ M arcos L uciano Pimenta PIN HEIROc
}

\begin{abstract}
RESUM 0
O bjetivou-se verificar e analisar junto à equipe de enfermagem o conhecimento sobre em que consiste um er ro de medicação, sua necessidade de notificação e o conteúdo da mesma. T rata-se de pesquisa de caráter quantitativo descritivo, desenvolvida em uma instituição hospital ar, da qual fizeram parte 72 profissionais. E $m$ relação à definição de erros de medicação, constatou-se que 49 (68\%) possuem conceito semelhante ao adotado nesse estudo. Quanto à necessidade de notificação, 67 (93\%) afirmaram sua necessidade. Referente ao conteúdo da notificação, 63 (85\%) possuem conceito semelhante ao adotado no estudo. Evidencia-se a necessidade de aprofundamento em relação aos aspectos referentes aos erros de medicação.
\end{abstract}

D escritores: Sistemas de medicação. Veícul os homeopáticos. E quipe de enfermagem.

\section{RESUMEN}

E I objetivo fue verificar y analizar con el equipo de enfermería el conocimiento sobre en quéconsiste un er ror de medición, la necesidad de ser notificado y lo que debe constar en dicha notificación. Consi ste en una investigación cuantitativa descriptiva, desarrollada en una institución hospitalaria, dela cual formaron parte 72 personas. E $n$ relación a la definición de er rores de medición, 49 (68\%) tienen concepto semejanteal adoptado en este estudio. E n cuanto a la necesidad de notificación, 67 (93\%) afirmaron que existe dicha necesidad. E n relación al contenido de la notificación, 63 (85\%) tienen concepto semejante al adoptado en este estudio. F ue evidente la necesidad de profundizar en relación a los er rores de medicación.

D escriptores: Sistemas de medicación. Vehículos homeopáticos. G rupo de enfermería.

Título: E rrores de medición: análisis del conocimiento del equipo de enfermería de una institución hospitalaria.

\section{ABST RACT}

Theaim of this study was to deter mineand discuss with the nursing staff their know ledgeabout what represents a medication error, theneed to notify such an er ror and what w ould benecessary to put in thesenotifications. This is a descriptivequantitative study, carried out in a hospital, of which 72 professionals took part. R egarding the definition of medication er rors, wefound that $49(68 \%)$ sharethe concept adopted in this study. R egarding the need of notification, $67(93 \%)$ declared that thereis such a need. As regards the content of the notification, $63(85 \%)$ had a similar concept to the one used in this study. The need to further approach aspects related to medication er rors was evident.

D escriptors: M edication systems. H omeopathic vehicles. N ursing, team.

T itle: M edication er rors: analysis of what a hospital's nursing staff know.

\footnotetext{
a D outor em Enfer magem, Professor A djunto III do D epartamento de Enfermagem da U niversidade Federal dos Vales do Jequitinhonha e M ucuri (UFVJM), Diamantina, M inas G erais, Brasil.

- A cadêmico do 8o Período do Curso de G raduação em Enfermagem da U FVJM, Diamantina, M inas Gerais, Brasil.

c D outor em Farmácia, Professor A djunto III do D epartamento de Ciências Básicas da U FVJ M , Diamantina, M inas G erais, Brasil.
} 


\section{INT RODUÇÃO}

Os erros de medicação no processo da administração de medicamentos têm sido identificados em vários estudos nacionais e internacionais, apresentando frequência de 14,9 a $59,5 \%(1,2)$.

Entre os fatores que ocasionam tais erros, destacam-se: letra ilegível ou ambígua na prescrição médica, separação pela farmácia de medicamento diferente da prescrição, erro na via de administração do medicamento e inter ação medicamento$\mathrm{sa}^{(3)}$.

Outros estudos relatam que os erros de medicação são sistêmicos e citam diversos fatores como contribuintes, tais como a via de administração, a complexidade exigida para o procedimento, as características farmacológicas e o conhecimento da equipe de enfermagem em relação ao medicamento $0^{(4,5)}$.

Vale destacar que, como as etapas finais do sistema de medicação são atribuições da equipe de enfermagem, portanto, cabe a ela a última oportunidade de interceptar o erro de medicação. Somase a isso o fato de as fal has nos processos de preparo e administração serem fatores que comprometem a eficácia no sistema de medicação e, consequentemente, a excelência que as instituições de saúde devem oferecer à sociedade ${ }^{(6)}$.

Destaca-se ainda, no que diz respeito aos erros de medicação, a presença da subnotificação ou a não notificação devido ao medo e ao receio presentes em tais situações. 0 desconhecimento do que seja efetivamente um erro, o desconhecimento perante as intercorrências com o paciente e o temor quanto ao seu futuro profissional, também são fatores que levam a equipe de enfermagem a não relatar o erro de medicação(7).

A ssim, devido à importância da temática "erro de medicação" e à exigência pelos programas de acreditação hospitalar, o desenvolvimento e implementação de protocolos e formulários de notificação apresentam-se como estratégias para medir e monitorar os erros de medicação e o desempenho dos serviços hospitalares, possibilitando uma análise crítica sobre as causas de sua ocorrência e implantação de medidas de qualidade ${ }^{(8)}$.

0 objetivo para esse estudo foi verificar e analisar junto à equipe de enfermagem o conhecimento sobre em que consiste um erro de medicação, sua necessidade de notificação e o conteúdo da mesma.

\section{MÉTODOS}

T rata-se de um estudo quantitativo descritivo, realizado em uma instituição hospitalar beneficente de um município do interior do estado de $M$ inas $G$ erais. Dentre as especialidades de atendimento de saúde observadas estão a clínica médica, a neurologia e a cirurgia geral.

A amostra foi escolhida por conveniência e teve como critérios de inclusão a disponibilidade dos profissionais para responderem ao questionário e sua participação ativa no preparo e administração de medicamentos.

A ssim, o estudo objetivou 0 al cance da total idade, ou seja, 97 sujeitos da equipe de enfermagem, pois todos atuam diretamente na administração de medicamentos. No entanto, a amostra constitui-se de 72 profissionais: 9 enfermeiros, 46 técnicos de enfermagem e 17 auxiliares de enfermagem, uma vez que 25 profissionais recusaram-se a participar da pesquisa.

A coleta de dados foi realizada através da aplicação de um questionário, o qual foi adaptado de um estudo consagrado pela liter atur $\mathrm{a}^{(9)}$, fornecido para os sujeitos da pesquisa, após explicação e esclarecimento de dúvidas pelo pesquisador.

Foi estipulado um prazo de três dias para o preenchimento e devolução do instrumento de coleta de dados, de 19 a 22 de abril de 2010. 0 questionário contemplou dados referentes ao gênero, faixa etária, categoria profissional, tempo de profissão, carga horária, turno e questões sobre em que consiste um erro de medicação, sua necessidade de notificação e dados necessários nessa notificação.

$\mathrm{Na}$ apresentação dos resultados e discussão foi realizada a caracterização demográfica da amostra e anál ise das respostas, utilizando-se a estatística descritiva.

0 projeto de pesquisa teve a aprovação do Comitê de Ética em Pesquisa da U niversidade Estadual de M ontes Claros, sob o processo de no 1789/2009, e o consentimento da direção da instituição hospitalar pesquisada. Os princípios éticos foram seguidos de acordo com a Resolução 196/ 96 do Conselho N acional de Saúde ${ }^{(10)}$.

\section{RESULTADOS E DISCUSSÃO}

$\mathrm{N}$ um total de 72 participantes, encontrou-se 56 (78\%) do gênero feminino e 16 (22\%) do gênero 
masculino. Quanto à faixa etária, 12 (17\%) estão entre 20 e 25 anos, 10 (14\%), entre 26 e 30 anos, 15 (21\%), entre 31 e 35 anos, 21 (29\%), entre 36 e 45 anos, 8 (11\%), entre 46 e 50 anos e $6(8 \%)$, entre 51 e 55 anos. Portanto, apresentam mediana de 33, limite superior de 55 e limite inferior de 20.

Quanto à categoria profissional, 9 (12\%) são enfermeiros, 17 (24\%), auxiliares de enfermagem e $46(64 \%)$, técnicos em enfermagem. Constatou-se que 23 (32\%) possuem tempo de profissão entre 1 e 5 anos, 13 (18\%), entre 6 e 10 anos, 29 (40\%), entre 11 e 20 anos e 7 (10\%), entre 21 e 30 anos, apresentando mediana de 10, limite superior de 30 e limite inferior de 01. Quanto à carga horária de trabal ho, 58 (80\%) trabal ham 48 horas, 7 (10\%), 44 horas e 7 (10\%), 40 horas semanais. A respeito do turno de trabal ho, 40 (56\%) pertencem ao diurno e 32 (44\%) ao noturno. D estacam-se, portanto, quantitativamente, os técnicos de enfermagem, na categoria entre 06 a 10 anos de profissão, trabalhando 48 horas semanais e em turno diurno.
Os dados a seguir, contidos na T abela 1, referem-se às respostas fornecidas pela equipe de enfer magem em relação à definição de erro de medicação, classificadas como idêntica, semelhante e discordante, reportando-se à definição do $\mathrm{N}$ ational Coordinating Council for M edication Error Reporting and $P$ revention (N CCMERP), a qual se segue: "qualquer evento passível de prevenção que pode causar ou induzir ao uso inadequado do medicamento ou prejudicar o paciente enquanto o medicamento está sob o controle do profissional de saúde, paciente ou consumidor. T ais eventos podem estar relacionados à prática profissional, produtos de cuidado de saúde, aos procedimentos e sistemas, incluindo prescrição; à comunicação da prescrição; ao rótulo do produto, à embalagem e nomenclatura; à composição; à dispensação; à distribuição; à educação; à monitoração e ao uso"(11).

Procedeu-se também o agrupamento de respostas semel hantes para melhor visualização dos dados, o que pode ser observado, através da T abela 1.

T abela 1 - Distribuição das respostas referentes à definição de erro de medicação. Diamantina, M G, 2010.

\section{Respostas}

$\mathrm{N}$ ão ler a letra do médico. Prescrições com letra difícil.

$\mathrm{N}$ a hora da diluição, ser administrado por ordem verbal, sem segurança para o funcionário

M edicação trocada de um paciente para o outro, ou uma medicação a mais ou a menos. Administrar medicamento em via ou paciente er rado

F alta de atenção, cansaço, pressa, estresse, sobrecarga, erro de far mácia

$\mathrm{N}$ ão ol har os dados vitais antes de administrar a medicação

A dministr ação de medicação sem verificar a prescrição médica

Fal ha cometida que poderá ou não prejudicar a saúde do paciente. Pode ocorrer na prescrição (médico), transcrição e administração (técnico de enfermagem), distribuição (farmácia) e outros

É transtorno tamanho que pode levar a óbito

A imperícia na administração não respeitando cliente, leito, horário, medicação e via

E rro ocorrido nas fases de administração, identificação, prescrição, dispensação, preparação e administração

A dministr ação errada de al gum fármaco (dosagem, paciente, horário ou medicação)

A dministração inadequada de medicação no paciente que pode levá-lo a morte Consiste na administração adversa aos 5 certos
Semelhante Discordante

n (\%) n (\%)

$10(13,88)$

$3(4,16)$

$1(1,38)$

$1(1,38)$

T otal

Fonte: Santa Casa de Caridade de Diamantina, M G, 2010.

De acordo com a Tabela 1, identifica-se a percepção do conhecimento da equipe de enfermagem sobre o tema e seu real conhecimento, quando a maioria das respostas, 49 (68\%), possui uma de- finição considerada semel hante acerca da definição de um erro de medicação. O bserva-se ainda, 23 (32\%) de definições discordantes. D estaca-se também 0 fato de nenhum profissional possuir uma 
definição idêntica ao N CCM ERP(11), entretanto, apresentam definições lógicas e relevantes acerca do motivo da ocorrência dos erros, apontando subsídios para a possibilidade de aperfeiçoar a educação e consequentemente colaborar com a minimização de erros.

Evidencia-se a quarta resposta, classificada como discordante, uma vez que falta de atenção, cansaço, pressa, estresse e sobrecarga são fatores causais do erro de medicação e não a sua definição. Há de se considerar a possibilidade de 0 questionário não ter sido adequadamente compreendido pelos participantes do estudo. A inda assim, surge uma questão importante quando se trata do tema erro de medicação: as condições de trabalho enfrentadas pela equipe de enfermagem que contribuem para a ocorrência dos mesmos.

T ambém é importante considerar que um dos obstáculos em relação ao estudo e à prevenção de erros de medicação é a falta de padronização e a multiplicidade da terminologia utilizada para sua classificação(12). Esta situação prejudica a comparação entre os estudos e retarda o conhecimento epidemiológico sobre tema de tão importante destaque ${ }^{(13)}$. A Organização M undial da Saúde (OMS) está buscando uma taxonomia internacional para erros de medicação, contudo, ela ainda não foi concluída(14).

Quanto à educação em serviço, extremamente necessária nesse contexto, é preciso lembrar que os projetos necessitam estar em consonância com os interesses dos envolvidos, atenderem aos anseios e às necessidades daqueles que vão participar, aos objetivos da instituição e, no caso da enfermagem, à finalidade do trabalho, que é a maximização da assistência, e propiciar à equipe conhecimentos sólidos sobre a temática erro de medicação(15).

T ais fatos nos reportam à reflexão da importância do conhecimento técnico-científico da equipe de enfer magem e atualização constante deste conhecimento, sendo a educação em serviço um influenciador da redução da ocorrência de erros previsíveis.

Outro aspecto tratado no estudo refere-se à necessidade da notificação perante a ocorrência de um erro. Do total, 67 (93\%) afirmam a necessidade de se notificar. A credita-se que os profissionais que não fazem parte desse quantitativo poderiam justificar tal afirmativa em razão do temor de advertências e até mesmo demissão, fato que, infelizmente, ocorre em al gumas instituições.
E sses resultados estão em concordância com um estudo semel hante que demonstrou que $87,3 \%$ da equipe de enfermagem deixam claro a necessidade de se notificar um erro de medicação e que $70,1 \%$ afirmam que as razões para a não notificação dos erros estão relacionadas ao temor da reação dos enfermeiros responsáveis ou colegas de trabalho(7).

M uitas instituições, após a ocorrência de erros de medicação, aplicam advertências aos profissionais que os cometeram. As advertências são vistas como uma forma de punição, o que ocasiona medo, pedido de demissão, sentimento de culpa e preocupações relacionadas à gravidade do erro, isso pode levar o indivíduo a não relatar seus erros e esse fato propicia consequências devastadoras não só para os pacientes, como também para os profissionais envolvidos ${ }^{(16)}$.

A lém disso, é necessário registrar a falta de estrutura de algumas instituições para gerenciar a ocorrência de um erro. Confirma-se também a resistência dessas instituições em admitir a existência do erro, o que faz com que não haja a notificação, a definição e a execução de estratégias para evitá- $\mid 0^{(3)}$, tampouco uma avaliação reflexiva e criteriosa acerca de sua magnitude ${ }^{(17)}$.

E $m$ vista disso, o NCCM ERP, acredita que os profissionais da saúde e as organizações devem ser incentivados a apresentar notificações de erros, buscando avaliá-los e preveni-los, bem como partiIhar experiências com seus pares, devendo-se criar também uma cultura não punitiva, fornecer a confiabilidade adequada, proteções legais e propiciar a aprendizagem sobre erros e suas soluções aos profissionais ${ }^{(18)}$.

No que diz respeito ao conteúdo dessa notificação, vale salientar o Formulário E rro de M edicação utilizado pela A gência $\mathrm{N}$ acional de Vigilância Sanitária (ANVISA). Segundo esse formulário, que possui caráter confidencial, o notificador deve informar data, hora e descrição/ sequência do evento, tipos de profissionais e serviços envolvidos, se o erro afetou o paciente, descrição da evolução do paciente, indicação das causas do erro, dados do paciente, local do erro, medicamentos envolvidos, recomendações para prevenção, estratégias e procedimentos tomados pela instituição. Por fim, temse o campo de identificação do notificador que é opcional ${ }^{(19)}$.

A seguir, na T abela 2, apresenta-se a distribuição das respostas fornecidas pela equipe de en- 
fermagem referente ao conteúdo da notificação de um erro de medicação, reportando-se à recomendação da AN VISA ${ }^{(19)}$. Procedeu-setambém o agru- pamento de respostas semelhantes para melhor visualização dos dados.

T abela 2 - Distribuição das respostas referentes ao conteúdo da notificação de um erro de medicação. Diamantina, M G, 2010.

\section{Respostas}

Semelhante n (\%)

M edicação, dose, via, horário e paciente. Avisar o médico

Explicar o que aconteceu, com qual medicação

A medicação administrada, os dados do paciente, patologia, prognóstico, dose administrada e condutas tomadas pelo profissional

N ome do profissional, qual erro cometeu, data, hora, local e cuidados prestados para minimizar as reações caso existam

Todas as intercorrências que ocorre com o paciente e medicação

Nome do cliente, medicação, médico e profissional que administrou

A medicação, o tipo de erro, as alterações e interações medicamentosas das quais o paciente sofreu

N ome do paciente, nome do profissional, qual medicação, via, dosagem, tipo de erro

N ome completo do paciente, procedimento exercido, horário, ocorrências, evolução com letra legível e assinatura do funcionário de cada horário

A hora, o paciente, a dosagem, a medicação, o motivo, a comunicação ao enfermeiro e médico

Todas as informações relacionadas ao evento, hora da ocorrência, em que consistiu o erro, repercussão clínica e causas possíveis

M edicação, horário, dosagem e paciente

D ata, horário, nome do paciente, profissional que cometeu o erro, consequência para o paciente e medidas tomadas pelo hospital

\section{T otal}

Fonte: Santa Casa de Caridade de Diamantina, M G, 2010.

N o que se refere ao conteúdo da notificação, $63(85 \%)$ responderam de forma semelhante ao que deve conter. Entretanto, vale ressaltar que $9(15 \%)$ não responderam, motivo pelo qual o quadro acima apresenta total de $85,0 \%$ e que não houve resposta idêntica aquela recomendada pela AN VISA ${ }^{(19)}$.

A qui, também, vale destacar que, embora não tenha havido respostas idênticas à recomendação em questão, depreende-se da T abela 2 que os indivíduos apresentam respostas coerentes. É preocupante, entretanto, o quantitativo daquel es que não responder am, atitude que pode ser justificada pela falta de conhecimento.

N esse cenário, é imperiosa a afirmação de que, para que os profissionais possam realizar a correta notificação, é necessária a orientação contínua, bem como 0 aprimoramento da educação da equipe de enfermagem. I sso se mostra como uma atitude de extrema importância, principalmente quando é voltada para o exame, e a diminuição/ elimi- nação das dúvidas existentes durante o preenchimento da notificação do erro(8).

E ssas orientações devem ser fornecidas pelo enfermeiro que necessita também realizar a supervisão como forma de minimizar os erros durante as notificações, sendo de extrema necessidade possuir amplo conhecimento em relação à temática "erros de medicação", buscando assim a confiança da equipe de enfermagem, uma prática humanizada e fundamentada cientificamente.

\section{CONSIDERAÇÕES FINAIS}

Este estudo possibilitou verificar e analisar o conhecimento da equipe de enfermagem sobre em que consiste um er ro de medicação, sua necessidade de notificação e conteúdo. Porporcionou-se, assim, uma análise crítica sobre o conhecimento e uma reflexão acerca da importância do aprimoramento da educação da equipe. 
Verificou-se que, embora a equipe não tenha apresentado definições idênticas àquelas do NCCM ERP(11), apresentou definições lógicas e relevantes. 0 mesmo ocorreu com a maioria das respostas referentes à necessidade de se notificar um erro, bem como ao conteúdo da notificação. Entretanto, é necessário atentar para as respostas discordantes em relação à definição e para a ausência de respostas em relação ao conteúdo da notificação.

D estaca-se, então, a educação em serviço como forma de se garantir um maior conhecimento técnico-científico e atualização constante da equipe de enfermagem. Espera-se que este estudo contribua para alertar sobre a importância do conhecimento da equipe de enfermagem sobre os erros de medicação, tornando-a uma influenciadora na redução de sua ocorrência e possibilitando uma correta notificação caso o erro aconteça. E sse conhecimento garante uma maior qualidade da assistência, a confiança da equipe e uma prática humanizada e fundamentada cientificamente.

É importante frisar que este estudo servirá de base para que outras pesquisas sejam realizadas sobre o tema, auxiliando as instituições e suas equipes a realizarem o enfrentamento necessário a evitar as iatrogenias causadas por erros de medicação e notificações não realizadas ou realizadas de forma incompleta.

\section{REFERÊ NCIAS}

1 Costa L A . Avaliação da administração de medicamentos em hospital público e privado de Salvador [ dissertação] . Salvador: F acul dade de M edicina, U niversidade Federal da Bahia; 2005.

$2 \mathrm{M}$ aricle $\mathrm{K}, \mathrm{W}$ hitehead $\mathrm{L}, \mathrm{R}$ hodes $M$. Examining medication errors in a tertiary hospital. J N urs Care Qual. 2007;22(1):20-7.

3 Praxedes M FS, Telles F ilho PCP. I dentificação de erros no preparo e administração de medicamentos pela equipe de enfermagem e das ações praticadas pela instituição hospitalar. Cogitare E nferm. 2008;13(4):514-9.

4 Fry M M , Daceep C. Factors contributing to incidents in medicine administration: part 1. Br J N urs. 2007; 16(9):556-8.

5 T ang FI, Sheu SJ, Yu S, W ei LL, Chen CM . Nurses relate the contributing factors involved in medication errors. J Clin N urs. 2007;16(3):447-57.
60 pitz SP. Sistema de medicação: anál ise dos er ros nos processos de preparo e administração de medicamentos em um hospital de ensino [ tese] . Ribeirão Preto: E scola de Enfermagem de Ribeirão Preto, U niversidade de São Paulo; 2006.

7 Bohomol E, Ramos LH. Erro de medicação: importância da notificação no gerenciamento da segurança do paciente. Rev Bras Enferm. 2007;60(1):32-6.

8 Silva BK, Silva JS, G obbo AFF, M iasso AI. Erros de medicação: condutas e propostas de prevenção na perspectiva da equipe de enfermagem. Rev Eletrônica Enferm. 2007;9(3):712-23.

9 Bohomol E, Ramos LH. Percepções sobre o erro de medicação: análise de respostas da equipe de enfermagem. Rev L atino-Am E nfermagem. 2006;14(6):63-8.

10 M inistério da Saúde (BR ), Conselho N acional de Saúde. Resolução 196, de 10 de outubro de 1996: diretrizes e normas regulamentadoras de pesquisa envolvendo seres humanos. Brasília (D F ); 1996.

$11 \mathrm{~N}$ ational Coordinating Council for M edication Error Reporting and Prevention. Taxonomy of medication errors [ Internet] . Rockville; 1998 [ cited 2010 A pr 14] . Available from: http:/ / www.nccmerp.org/ aboutM edE rrors.html.

12 A nacleto TA, Rosa M B, M eiva H M , M artins M AP. E rros de medicação. Pharm Bras. 2010;74(1):1-23.

13 Yu KH, Nation RL, D ooly M J. M ultiplicity of medication safety terms, definitions and functional meanings: when is enough? Qual Saf $\mathrm{H}$ ealth Care. 2005;14(5):358-63.

14 World Health Organization. World alliance for patient safety taxonomy [ I nter net] . G eneva; 2011 [ cited 2010 A pr 12] . Available from: http:/ / www. who.int/ patientsafety/ taxonomy.

15 Paschoal AS, M antovani M F, M éier M J. Percepção da educação permanente, continuada e em serviço para enfermeiros de um hospital de ensino. Rev EsC Enferm U SP. 2007;41(3):478-84.

16 H oefel H H K , M agal hães AM M , F alk M LR. A nálise das advertências geradas pelas chefias de enfermagem. Rev G aúcha Enferm. 2009;30(3):383-9.

17 Oliviera RC, M iasso AI, Cassiani SH B. Setor de emergência: situações de erros na medicação. In: Telles Filho PCP, Stuchi RAG, organizadores. A enfermagem no novo milênio: uma abordagem multidisciplinar. Belo H orizonte: D ifusora; 2008. p. 96-106. 
$18 \mathrm{~N}$ ational Coordinating Council for M edication Error Reporting and Prevention. Supports principles for patient safety reporting programs [I nternet]. Rockville; 2003 [ cited 2010 A pr 14] . Available from: http:// www.nccmerp.org/ press/ press2003-11- 25. html.
19 M inistério da Saúde (BR ), A gência N acional de Vigilância Sanitária, U nidade de Farmacovigilância. N otificação de suspeita de erro de medicação [ Internet]. Brasília (D F); 2010 [ citado 2010 abr 14] . D isponível em: http:/ / www.anvisa.gov.br/ servicos/ form/ farmaco/ erro de medicacao.pdf.
Endereço do autor / Dirección del autor /

\section{Author's address:}

Paulo Celso Prado T elles Filho

Rua Prof. Paulino G uimarães J únior, 160, ap. 03,

Centro

39100-000, D iamantina, M G

E-mail:ppradotelles@yahoo.com.br
Recebido em: 14/ 09/2010

A provado em: 03/ 05/ 2011 